\title{
Transfert rythmique du chinois mandarin au français dans l'apprentissage du Français Langue Étrangère : acoustique et perception
}

\author{
Claire Pillot-Loiseau ${ }^{1}$, et Han Xie ${ }^{1}$ \\ ${ }^{1}$ Laboratoire de Phonétique et Phonologie UMR 7018, Université Sorbonne Nouvelle, 19 rue des \\ Bernardins, 75005 Paris, France
}

Résumé. Cette étude concerne le transfert rythmique du chinois mandarin au français dans le cadre de l'apprentissage du Français Langue Étrangère. Le rythme de phrases françaises produites par dix apprenants sinophones de niveau de français intermédiaire-avancé, est comparé avec celui de leurs productions en chinois mandarin et celui en français produit par des natives. Acoustiquement, les mesures quantitatives du rythme mettent en lumière la différence significative entre les patterns rythmiques du chinois mandarin et du français produit par les sujets sinophones. Les valeurs des mesures rythmiques appliquées au français langue étrangère sont plus proches de celles appliquées au français natif, ce qui est en contradiction avec les résultats de l'étude perceptive selon lesquels le transfert rythmique du chinois mandarin au français peut être identifié par huit locuteurs natifs $\mathrm{du}$ français. La disparité entre acoustique et perception met en doute l'emploi de ces mesures dans cette étude sur le transfert rythmique.

\begin{abstract}
Rhythmic transfer from Mandarin Chinese to French in the learning of French as a foreign language: acoustics and perception. This study concerns the rhythmic transfer of Mandarin Chinese to French in the context of French as a Foreign Language learning. The rhythm of French spoken sentences produced by ten Chinese-speaking learners at an intermediate-advanced French level, is compared with that of their Mandarin Chinese productions, and with the rhythm of the same French sentences produced by native subjects. Acoustically, quantitative measures of rhythm show significant differences between the rhythmic patterns of Mandarin Chinese and French produced by Chinese subjects. The values of rhythmic measures applied to French as a foreign language are closer to those applied to native French, which is in contradiction with the results of the perceptual study according to which the rhythmic transfer of Mandarin Chinese to French can be identified by eight native speakers of French. The disparity between acoustics and perception casts doubt on the use of rhythmic measures in this rhythmic transfer study.
\end{abstract}




\section{Introduction}

« Nous avons de nombreuses preuves que, lors de l'apprentissage d'une langue étrangère, nous transférons tout notre système de langue maternelle dans le processus : [...] nos phonèmes et leurs variantes, nos schémas d'accentuation et de rythme, nos transitions, nos schémas d'intonation et leur interaction avec d'autres phonèmes » (Lado, $1957: 102)$. De nombreuses études se sont consacrées à la nature de ces transferts sur le plan segmental, mais, dans la mesure où le rythme structure le flux de parole et sert de repère dans la structuration des informations en communication orale, il nous a paru pertinent d'y consacrer cet article dans le cadre de l'apprentissage du Français Langue Etrangère $\left(\mathrm{FR}_{\mathrm{LE}}\right)$ par des sujets sinophones. Notre étude acoustique du rythme viendra quantifier le transfert rythmique du chinois mandarin au français, complétée par notre étude perceptive.

\section{Transfert phonétique de la langue maternelle dans l'apprentissage d'une langue étrangère}

Lorsque l'influence de la langue maternelle (LM) profite à l'apprentissage phonétique d'une langue étrangère (LE), le processus est nommé transfert (Ellis, 1994). En revanche, le terme d'interférence désigne le processus où la LM de l'apprenant entrainerait une production erronée en LE (Lado, 1957). Cependant, pour White \& Mattys (2007a, 2007b), le transfert peut aussi signifier un traitement faussé de la LE selon les règles sonores de la LM. Nous nous servons donc ici de transfert pour englober ces deux sens distincts.

\subsection{Aspect segmental}

Les études sur le transfert phonético-phonologique entre LM et LE abordent d'abord la notion de surdité phonologique évoquée en 1931 par Polivanov et développée par Troubetzkoy $(1938,1976)$. Le premier fait ressortir l'interférence phonologique de la LM du locuteur lors de l'écoute d'une langue autre que la sienne, alors que le second considère le système phonologique d'une langue donnée comme un crible à travers lequel passe tout ce qui est dit. Si un apprenant réalise mal un son de la LE, c'est parce qu'il l'a mal perçu et non pas à cause d'une incapacité articulatoire. Pourtant, la théorie du contrôle de la perception par l'action (Perception-for-Action-Control Theory: Schwartz et al. 2012) stipule que le locuteur-auditeur utilise une compétence sensorimotrice multimodale pour récupérer et traiter les indices acoustiques contenus dans le signal sonore, notamment au moyen du geste articulatoire.

En outre, il existe plusieurs niveaux de perception pouvant mettre en doute le transfert systématique des propriétés de la LM de l'apprenant vers la LE : un niveau dit «bas, périphérique » ou phonétique, et un niveau plus « élevé, central » ou phonologique (Borrell 1996). De ce fait, de nombreux modèles de perception phonétique interlangue ont pour base l'incorporation des caractéristiques phonético-articulatoires du segment et de sa fonction distinctive : le modèle d'assimilation perceptive (PAM) de Best (1995) et le modèle d'apprentissage de la parole (SLM) de Flege (1995) ont un point de départ similaire : les segments " nouveaux » d'une LE sont plus faciles à percevoir et acquérir que les segments similaires à ceux de la LM de l'apprenant en ce que les premiers permettent soit la création de nouvelles catégories pour la LE selon Flege (1995), soit la réorganisation des catégories perceptives pour la LM selon Best (1995). Dans le cadre du modèle d'effet magnétique de perception (PME) (Kuhl et al., 2008), les segments d'une LE ressemblant à un prototype phonétique de la LM du locuteur sont perceptivement attirés vers le prototype, d'où l'apprenant assimile ces segments analogues au prototype de la LM alors que la discrimination entre les segments 
étrangers perceptivement appartenant aux catégories distinctes dans sa LM serait plus facile pour lui.

\subsection{Aspect suprasegmental}

\subsubsection{Généralités}

Si les efforts ont été largement consacrés aux études sur la perception interlangue au niveau segmental, il en est autrement pour l'aspect suprasegmental (Rasier \& Hiligsmann, 2007). Borrell et Salsignac (dans Renard, 2002) agrandissent le champ de la notion de crible phonologique en précisant que celui-ci «engloberait un crible phonético-phonologique et un crible prosodique " (Renard, 2002). Celui-ci empêcherait la perception de la langue cible au niveau de la prosodie chez l'apprenant autant que le crible phonético-phonologique l'empêche sur le plan segmental. Dès lors, nous avons le droit de nous attendre au transfert des habitudes prosodiques de la LM de l'apprenant à la langue apprise.

\subsubsection{Transfert interlangue sur le plan rythmique}

Le rythme «conditionne la structuration temporelle sans laquelle il n'est pas de maitrise possible de la parole » des LE (Renard, 1979) : une bonne maitrise de l'aspect temporel d'une langue favorise l'intercompréhension entre interlocuteurs et ceux-ci tendent à considérer ce genre de production plus agréable et plus naturelle (Lai, 2011). Néanmoins, l'acquisition du rythme attire moins d'attention que les aspects intonatif et accentuel concernant l'apprentissage phonétique des LE (Wenk, 1985).

Selon Wenk (1985), si les patterns rythmiques de la LM et de la LE de l'apprenant ne se rapprochent pas les uns des autres, il se peut que celui-ci perçoive et produise le rythme étranger comme celui de sa LM. En effet, l'anglais à Singapour et en Malaisie témoigne du transfert rythmique de certains dialectes au sud de la Chine: la rythmicité syllabique résultant du transfert est mise en évidence soit par les résultats perceptifs dans Brown (1988), soit par les données acoustiques dans Low et al. (2000). Mok \& Dellwo (2008) trouvent que l'anglais produit par des natifs du cantonais et du chinois mandarin est perçu comme ayant un rythme syllabique par des natifs de l'anglais, contrairement à certains résultats des mesures quantitatives objectives du rythme qui indiquent la rythmicité accentuelle des paroles en anglais produites par des natifs du cantonais et du chinois mandarin, deux langues dont le rythme est estimé syllabique.

Qu'en est-il du transfert rythmique entre deux langues qui sont proches au niveau rythmique ? Gut (2003) étudie aussi bien les productions de laboratoire que les paroles spontanées d'apprenants étrangers de l'allemand: d'après ses données acoustiques, le rythme de leur production en allemand ressemble davantage à celui de leur LM, quelle que soit la classe rythmique à laquelle appartient leur LM. Cela dit, la conclusion de Wenk (1985) mentionnée plus haut pourrait aussi être appliquée au cas où les patterns rythmiques de la LM et de la LE se ressemblent les uns aux autres. Pourtant, le "décalage » entre perception et acoustique n'est plus à démontrer (Mok \& Dellwo, 2008; White \& Mattys, 2007b). Le transfert rythmique mérite, de ce fait, d'être envisagé d'un point de vue aussi bien instrumental que perceptif. Pour ce faire, nous allons aborder, dans la section suivante, la typologie rythmique et les mesures acoustiques permettant de classifier les rythmes linguistiques.

\section{Typologie rythmique et mesures acoustiques du rythme}




\subsection{Définition du rythme linguistique et dualité rythmique}

Sa notion recouvre une telle multitude de réalités (Astésano, 2001) que toute définition simple et précise du rythme semble être impossible pour certains auteurs (Fraisse, 1956). Structure des intervalles dans la succession d'évènements qui se caractérisent par leur périodicité (Allen 1972, cité par Mairano, 2010), le rythme est aussi défini par l'impression subjective créée par des unités qui se répètent à intervalles réguliers (Ramus 1999a).

Cependant, la dualité rythmique n'existe pas uniquement entre les réalités acoustique et perceptive, mais également entre une structure temporelle et une structure intensive et/ou harmonique (Astésano, 2001). Selon Fraisse (1956) et Allen (1972, dans Mairano, 2010), le rythme aurait à la base une structure temporelle sur laquelle se développeraient les variations intensives et tonales. Bien que Dell (1984, dans Astésano, 2001) mette en avant les schèmes accentuels qui, selon lui, constituent seuls la structure rythmique, la structuration de durée sert également à le définir. La hiérarchisation de deux structures du rythme va donc à l'encontre de sa conception unificatrice. Astésano (2001) propose donc de considérer le rythme comme «l'organisation temporelle des proéminences » comme dans Di Cristo \& Hirst (1997). Di Cristo $(2013,2016)$ trouve que «le rythme linguistique se construit sur une alternance plus ou moins régulière de temps forts et de temps faibles, les temps forts étant assimilables, dans la parole, à des syllabes accentuées, et les temps faibles, à des syllabes inaccentuées », ce qui permet de "dépasser la dichotomie entre "ordre temporel » et «ordre accentuel» (Astésano, 2001). Dans le cadre de notre étude, nous définissons le rythme de la parole comme l'impression subjective créée par la récurrence des unités acoustiques similaires qui se distribuent au cours du temps de manière relativement isochronique (Grabe \& Low, 2002).

\subsection{Etudes empiriques sur la typologie rythmique des langues du monde}

Rappelons que les langues à isochronie syllabique (espagnol, français) possèdent des intervalles inter-syllabiques de durées comparables, alors que l'accentuation des langues à isochronie accentuelle (anglais) se répète régulièrement au cours du temps (Pike 1945, Abercrombie 1967). Dans les langues à isochronie moraïque (japonais, tamoul, Ladefoged, 1975; Ramus, 1999a et 1999b), la more assume le rôle d'unité rythmique et se répète à intervalles réguliers, faisant de ces langues des systèmes davantage similaires aux langues à isochronie syllabique (Grabe \& Low 2002). Toutefois, l'isochronie sur laquelle ces études se basent est mise en question dès les années 60 et la typologie rythmique des langues est reprochée d'être dépourvue d'arguments expérimentaux plus parlants que ceux de perceptions relativement personnelles (Grabe \& Low, 2002) et parfois illusoires : l'auditeur tend à percevoir l'isochronie inter-accentuelle bien que celle-ci n'existe pas vraiment au niveau acoustique (Roach 1982).

En outre, la typologie rythmique revêt certaines bases phonologiques : le rythme parlé n'est plus considéré comme cause « de l'organisation temporelle des langues, mais comme conséquence de certaines de leurs propriétés phonologiques [...] : complexité des syllabes, [...] présence ou non de réduction vocalique... » (Ramus, 1999b), d'où un continuum entre langues à isochronies syllabique et accentuelle (Dauer 1983).

\subsection{Mesures quantitatives objectives du rythme}

Dès la fin des années 90, les nouvelles approches sur le rythme de la parole concernent essentiellement les mesures quantitatives objectives du rythme des intervalles vocaliques, des intervalles consonantiques, ainsi que de leurs degrés de variabilité (Ramus et al. 1999). 


\subsubsection{Mesures des intervalles vocaliques/consonantiques non-normalisés}

Ces mesures ne mettent pas l'accent sur la durée des phonèmes individuels.

$\Delta \mathrm{V}$ mesure l'écart-type de la durée des intervalles vocaliques de l'énoncé et reflète la présence/absence de réduction vocalique dans une langue donnée : les langues autorisant la réduction vocalique (anglais) ont ainsi une valeur de $\Delta \mathrm{V}$ plus grande que les autres qui l'autorisent moins (français).

$\Delta \mathrm{C}$ mesure l'écart-type des intervalles consonantiques (successifs et non successifs) et reflète la complexité des structures syllabiques d'une langue. Sa valeur sera inférieure pour les langues comme le français dont les structures syllabiques sont assez simples.

$\% \mathrm{~V}$ mesure la proportion des intervalles vocaliques (non-normalisée) de l'énoncé (Ramus et al., 1999) et est l'indice acoustique de la complexité des structures syllabiques et de la présence/absence de réduction vocalique à la fois. L'espace bidimensionnel $\Delta \mathrm{C} / \% \mathrm{~V}$ reflète l'isochronie syllabique/accentuelle perçue dans différents groupes de langues comme l'illustre la figure 1 (gauche). Ces grandeurs sont censées prédire l'appartenance d'une langue donnée à telle ou telle classe rythmique (Ramus et al. 1999).
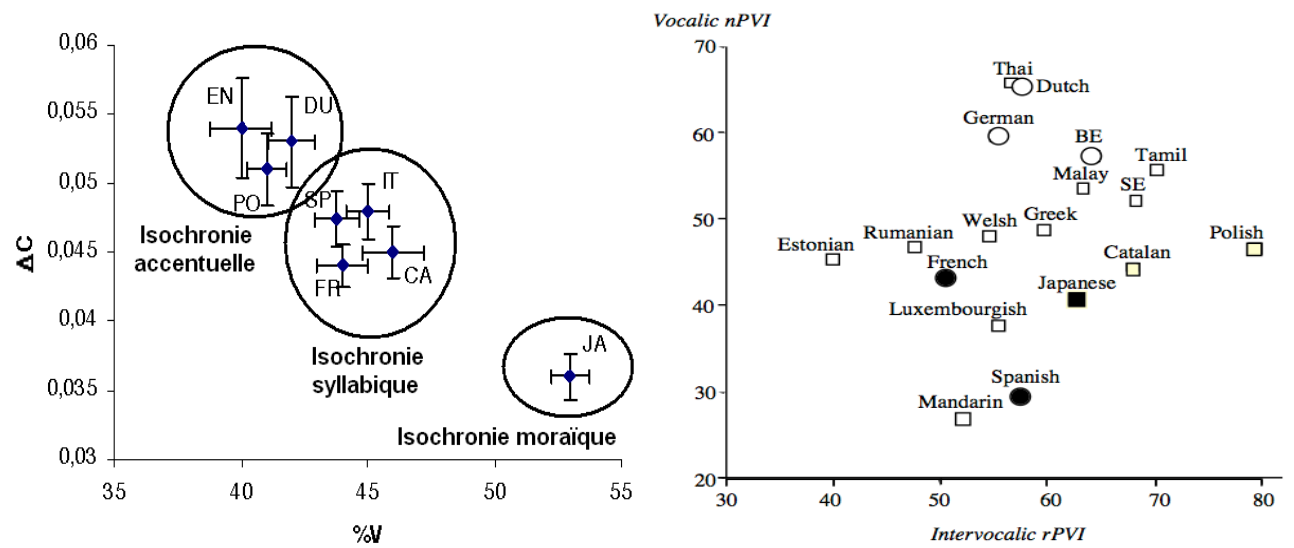

Fig. 1. Gauche : Moyennes et écart-types des intervalles consonantiques (y) et proportion des intervalles vocaliques (x) $\Delta \mathrm{C} / \% \mathrm{~V}$ (Ramus et al., 1999). CA : catalan, DU : néerlandais, $\mathrm{EN}$ : anglais, FR : français, IT : italien, JA : japonais, PO : polonais, SP : espagnol. Droite : Profils de 18 langues selon les indices de variabilités par paires (Grabe \& Low 2002). Cercles vides : langues à isochronie accentuelle, cercles pleins : isochronie syllabique; carrés pleins : isochronie moraïque; carrés vides : langues non classifiées rythmiquement/mixtes. BE : anglais britannique. SE : anglais de Singapour.

\subsubsection{Indices de variabilités par paires (PVI, Low et al. 2000; Grabe \& Low 2002)}

Ces mesures indiquent la relation entre le timing de la parole et la classification rythmique des langues. rPVI est la mesure des variabilités brutes des intervalles vocaliques/consonantiques successifs, et nPVI celle de ces variabilités normalisées. Les valeurs de nPVI sont plus élevées dans les langues à rythmicité accentuelle (figure 1, droite), et inférieures pour les langues de rythme syllabique et moraïque, car les intervalles vocaliques sont plus variés dans des langues à isochronie accentuelle. Toutefois, les langues non-prototypiques se montrent toujours difficiles à classifier par les PVIs. La distinction entre rythme accentuel et rythme syllabique est donc loin d'être catégorielle car les stimuli des langues à isochronie accentuelle sont souvent prononcés plus rapidement que ceux des langues à isochronie syllabique (Arvaniti, 2009).

\subsubsection{Mesure des intervalles vocaliques/consonantiques normalisés}

Pour tenir compte des tempi variés inter-locuteurs et inter-langagiers, Arvaniti (2009), Ramus (2002) et Dellwo (2006) proposent un coefficient de variation de l'écart-type des 
intervalles consonantiques $\Delta \mathrm{C}$, nommé Varco $\Delta \mathrm{C}$, afin de normaliser $\Delta \mathrm{C}$. Varco $\Delta \mathrm{C}$ s'obtient en multipliant $\Delta \mathrm{C}$ par 100 et en divisant cette valeur par la moyenne de la durée de chaque intervalle consonantique. Une normalisation analogue (Varco $\Delta C$ ) est effectuée pour l'écart-type des intervalles vocaliques ( $\Delta \mathrm{V}$, White \& Mattys, 2007a et 2007b).

Toutes les mesures précédemment décrites se complètent les unes les autres car on ne peut pas prédire la classe rythmique d'une langue par une seule mesure.

\subsubsection{Limites}

Parce que chaque sujet a une façon de parler qui lui est particulière, l'appréhension de la variation inter-sujet est problématique (Arvaniti, 2009; Mairano, 2010; Ramus, 2002). En outre, Grabe \& Low (2002) ne sollicitent qu'un locuteur pour chaque langue analysée. De plus, les productions utilisées dans des expériences différentes varient aussi bien en matière de style qu'en matière de structure syllabique (parole spontanée, récits, différentes longueurs de productions). Enfin, ces mesures rythmiques censées démontrer l'isochronie au niveau temporel, n'englobent pas le changement d'accentuation et de fréquence fondamentale, ce qui mérite d'être étudié de plus près par des moyens instrumentaux.

Malgré la mise en doute de ces métriques, il nous parait cependant intéressant de les appliquer à notre corpus en vue de mesurer le transfert rythmique du chinois mandarin au français. Les rythmes de ces deux langues sont d'abord étudiés dans la section suivante.

\section{Rythmes du français et du chinois mandarin}

Le chinois mandarin, langue chinoise commune officielle depuis 1955 en Chine continentale, fait ici référence à la prononciation pékinoise représentative du mandarin du Nord de la Chine (Duanmu 2007).

\subsection{Syllabe}

Dans la mesure où la syllabe, élément abstrait du langage oral, sert à analyser la structure des segments et la structure rythmique de la langue (Angoujard, 1997), il paraît ici indispensable d'indiquer quelques éléments relatifs à la structure syllabique des deux langues qui nous intéressent. Wioland (1991) trouve que 80,55\% des syllabes en français sont ouvertes et que 19,45\% d'entre elles sont fermées. En chinois mandarin, le ton est, entre autres, une composante indispensable à chaque syllabe. En outre, dans cette langue, il existe une coïncidence entre une syllabe, un idéogramme et un mot : chaque idéogramme $\mathrm{du}$ chinois mandarin ne contient qu'une syllabe donc il est une langue monosyllabique, ce qui le distingue nettement du français dont $90 \%$ des mots sont plurisyllabiques (Lété, 2008). Alors que le français peut présenter les principaux types syllabiques suivants (C pour consonne, et $\mathrm{V}$ pour voyelle) : $\mathrm{CV}, \mathrm{CCV}, \mathrm{CCCV}, \mathrm{CVC}, \mathrm{VC}, \mathrm{V}$, le chinois mandarin présente les principales catégories suivantes: V, VV, VVV, CV, CVV et CVVV. La plupart de ces syllabes sont donc simples dans les deux langues.

\subsection{Accentuation}

Le français standard (lecture non expressive ou parler neutre, Léon, 2011) est une langue à accentuation fixe : son accentuation tombe sur la dernière syllabe du groupe sémantique (oxytonique, Léon, 2011). Le principal marqueur accentuel est alors la durée. S'ajoute l'accentuation initiale de mot motivée par la longueur du mot, son poids sémantique et le style du discours. Elle est réalisée par une montée de fréquence fondamentale (F0, Duez, 
2007). Enfin, l'accentuation d'insistance permet une mise en relief d'une unité généralement plus petite qu'un groupe rythmique; elle se réalise souvent sur la première syllabe du groupe rythmique (Léon, 2011). La durée et l'intensité en sont, dans ce cas, les deux principaux paramètres perceptifs.

L'accentuation du chinois mandarin est mise en relief par un changement de durée, de hauteur et éventuellement d'intensité (Chao, 1968); l'accentuation normale assume la fonction démarcative et parfois la fonction distinctive et l'accentuation de contraste est équivalente à l'accentuation d'insistance française. Cependant, l'accentuation normale chinoise n'est pas fixe, comme d'autres langues à rythmicité accentuelle. Dans cette langue, il existe un phénomène de réduction vocalique qui aura lieu lorsque le ton sur la voyelle est neutralisé. La syllabe qui comprend cette voyelle est alors inaccentuée (Mok \& Dellwo, 2008; Mok, 2009), réduite et son timbre ressemblera à celui du schwa (Mok, 2009).

\subsection{Rythme}

Comme déjà évoqué, le français est décrit comme une langue à isochronie syllabique, ce qui est confirmé par les mesures instrumentales (Ramus et al. 1999; Grabe \& Low 2002). Cependant, Wenk \& Wioland (1982) ainsi que Lacheret-Dujour (Léon, 2011) contestent l'isochronie syllabique du français en le qualifiant de langue à queue accentuée.

Pour Cao (1991, 1995, dans Lai, 2011), le rythme du chinois mandarin se caractérise par l'allongement de la première syllabe et le raccourcissement de la dernière syllabe d'un groupe rythmique. Cela étant, le chinois mandarin tend à avoir un pied avec la tête à gauche comme l'anglais. D'un point de vue structural, Wang (2004) et Liu (2003) considèrent que le chinois mandarin n'est ni une langue à isochronie syllabique, ni une langue à isochronie accentuelle : l'intervalle entre syntagmes est relativement plus long et plus relâché que celui qui est intra-syntagmatique. La structuration rythmique du chinois mandarin est donc, selon eux, de type temporel et se caractérise par l'alternance de relâchement/resserrement. Les mesures instrumentales du rythme du chinois mandarin plaident en faveur d'une isochronie syllabique malgré la présence de réduction vocalique et d'une accentuation lexicale non fixe. C'est la raison pour laquelle le transfert rythmique du chinois mandarin au français nous parait un sujet intéressant à aborder.

\section{But de l'étude}

Le transfert rythmique du chinois mandarin au français est peu étudié (Gabriel et al. 2014), mais mérite de l'être, car ces deux langues participent à la même classe rythmique du point de vue perceptif et acoustique mais se distinguent l'une de l'autre en termes de propriétés phonologiques relatives au rythme. Notre objectif est d'investiguer le rythme des énoncés en français produits par des apprenants sinophones du $\mathrm{FR}_{\mathrm{LE}}$. Étant donné le « décalage » entre deux aspects - "réalité acoustico-phonétique et réalité subjective (perception)» (Astésano, 2001 : 125) - du rythme de la parole, il nous semble intéressant et nécessaire de procéder à une étude à la fois acoustique et perceptive. Acoustiquement, les mesures rythmiques serviront à quantifier l'organisation temporelle de la production de nos locuteurs. Concernant l'étude perceptive, notre étude se concentrera sur la perception par des français natifs du rythme de productions françaises de natifs du chinois mandarin. Finalement, nous tenterons de vérifier si les résultats au niveau acoustique et perceptif démontrent conjointement notre hypothèse sur le transfert rythmique.

Dans la mesure où les segments étrangers similaires à ceux qui existent déjà dans le système phonologique maternel du locuteur seraient assimilés à ces derniers (Best 1995, Flege 1995), nous supposons donc que les locuteurs natifs du chinois mandarin qui apprennent le $\mathrm{FR}_{\mathrm{LE}}$ produisent le rythme français avec un pattern semblable à celui de leur LM dans la mesure où ces deux langues appartiennent aux langues à rythmicité syllabique 
du point de vue perceptif et acoustique. De ce fait, acoustiquement, les valeurs des mesures appliquées à leurs productions en français langue étrangère $\left(\mathrm{FR}_{\mathrm{LE}}\right)$ sont supposées plus proches de celles de leurs productions en chinois mandarin langue maternelle $\left(\mathrm{CM}_{\mathrm{LM}}\right)$ que de celles des productions en français de natifs du français langue maternelle $\left(\mathrm{FR}_{\mathrm{LM}}\right)$. Perceptivement, nous nous attendons à ce que les auditeurs français natifs perçoivent le rythme des productions en $\mathrm{FR}_{\mathrm{LE}}$ comme celles en $\mathrm{CM}_{\mathrm{LM}}$ et non comme celles en $\mathrm{FR}_{\mathrm{LM}}$.

\section{Etude acoustique}

\subsection{Méthodologie}

Le profil des 10 sujets sinophones est détaillé dans le tableau 1. Cinq locuteurs (un homme et quatre femmes) natifs du français ont également participé à nos enregistrements, dont quatre étudiants en deuxième cycle universitaire.

Tableau 1. Profil des locuteurs natifs du chinois mandarin apprenants de $\mathrm{FR}_{\mathrm{LE}}$

\begin{tabular}{|c|c|l|c|c|c|}
\hline Sexe & Age & Cursus & $\begin{array}{c}\text { Niveau de français, } \\
\text { début et condition } \\
\text { de son apprentissage }\end{array}$ & $\begin{array}{c}\text { Séjour } \\
\text { en France } \\
\text { depuis ... }\end{array}$ & Autres langues parlées \\
\hline $1 \hat{\jmath}, 99$ & $\begin{array}{c}23-28 \text { ans } \\
(\mathrm{M}=25 ; \\
\mathrm{ET}=1,93)\end{array}$ & Bac+4 & $\begin{array}{c}\text { Au moins B2, } \\
\text { français appris depuis } \\
6 \text { années en moyenne, } \\
\text { en Chine }\end{array}$ & $\begin{array}{c}5 \text { sujets <3ans; } \\
5 \text { sujets } \\
\text { entre } 3 \& 6 \\
\text { ans }\end{array}$ & $\begin{array}{c}8 \text { sujets : dialectes chinois (même } \\
\text { groupe linguistique que mandarin) } \\
\text { - Anglais appris depuis 15 années } \\
\text { (B1/B2) }\end{array}$ \\
\hline
\end{tabular}

Dix phrases en français et dix phrases en chinois mandarin répétées une fois ont été enregistrées (tableau 2), avec un nombre de syllabes analogue (en français : 21,1;ET=1,91; en mandarin : 21,$5 ; \mathrm{ET}=0,97$ ) et sélectionnées aléatoirement pour obtenir un corpus aussi naturel que possible, avec une distribution équilibrée des segments et des accentuations. Le corpus en français est extrait et modifié des exercices de compréhension orale de deux livres sur l'entrainement à la langue française (CD audio inclus : Chevallier-Wixler et al. 2007; Kober-Kleinert et al. 2007). Nous avons donc, en amont, l'enregistrement de notre corpus en français produit par des locuteurs natifs du français, ce qui permet de constituer une partie des stimuli originaux dans le test de discrimination. Par conséquent, 100 phrases enregistrées en $\mathrm{CM}_{\mathrm{LM}}$ (10 sujets $\times 10$ phrases), 100 phrases enregistrées en $\mathrm{FR}_{\mathrm{LE}}$ (10 sujets $\times 10$ phrases $)$ et 50 phrases enregistrées en $\operatorname{FR}_{\mathrm{LM}}(5$ sujets $\times 10$ phrases $)$ ont été obtenues.

Tableau 2. Extrait du corpus utilisé pour les mesures acoustiques

\begin{tabular}{|c|c|c|}
\hline Transcription d'une phrase & $\begin{array}{c}\text { Transcription } \\
\text { phonologique API }\end{array}$ & $\begin{array}{l}\text { Nombre de } \\
\text { syllabes }\end{array}$ \\
\hline $\begin{array}{l}\text { Des «bien-logés » ont décidé de partager leur sort et } \\
\text { de coucher dans la rue. }\end{array}$ & $\begin{array}{c}\text { /de bjẽ loze / õ deside } \\
\text { də pastaze lœь səь / e } \\
\text { də kufe dã la вy// }\end{array}$ & 21 \\
\hline 很多风水先生专门测算建筑物和地形之间的关系。 & 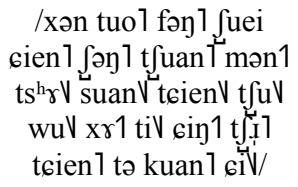 & 21 \\
\hline
\end{tabular}


Les sujets ont été enregistrés en chambre sourde (microphone Audio-technica AT8033, logiciel Pro Tools LE 7.4.2., fréquence d'échantillonnage : 44100Hz). Après une consigne donnée en LM, les sinophones ont, après une lecture mentale si besoin, lu les 10 phrases en mandarin dans un débit naturel, avec une pause entre chaque phrase. Puis il en a été de même pour les 10 phrases françaises, lues après écoute d'un extrait de média français de $2 \mathrm{mn} 18$, pour éviter que les sujets restent influencés par les consignes initiales données en mandarin. Les françaises ont lu de la même façon les phrases françaises. En moyenne, l'enregistrement des productions en $\mathrm{CM}_{\mathrm{LM}}$ a duré $82 \mathrm{~s}(\mathrm{ET}=15,3)$ par locuteur, celui en $\mathrm{FR}_{\mathrm{LE}}$ a duré $124 \mathrm{~s}(\mathrm{ET}=26,9)$ par locuteur et celui en $\mathrm{FR}_{\mathrm{LM}}$ a duré $73 \mathrm{~s}(\mathrm{ET}=12,6)$ par locuteur.

Après une annotation des fichiers en intervalles «silence » et "signal » grâce à la commande Intensity : To TextGrid (silences) du logiciel Praat (Boersma et Weenink, 2013), une autre tier a été générée avec l'annotation des intervalles vocaliques (V) et consonantiques (C) selon les critères de segmentation de Grabe \& Low (2002), Lin \& Wang (2007) et White \& Mattys (2007a), avec, en accord avec ces auteurs, la suppression des pauses à l'intérieur de ces phrases.

Après l'extraction de la durée des intervalles vocaliques et consonantiques de chaque production à l'aide d'un script Praat disponible en ligne ([http://celinedelooze.com/Homepage/Resources files/Get interval duration.praat] consulté le 20 avril 2014), six des mesures rythmiques déjà décrites ont été calculées à l'aide d'Excel pour chaque enregistrement de chaque locuteur (écart-type des intervalles consonantiques de l'énoncé $\Delta \mathrm{C}$, proportion des intervalles vocaliques de l'énoncé $\% \mathrm{~V}$, coefficient de variation du $\Delta \mathrm{C} \operatorname{Varco} \Delta \mathrm{C}$, coefficient de variation du $\Delta \mathrm{V} \operatorname{Varco} \Delta \mathrm{V}$, variabilité brute des intervalles consonantiques successifs rPVI-C et variabilité normalisée des intervalles vocaliques successifs nPVI-V). Un test $\mathrm{t}$ indépendant sur chaque mesure a été effectué à l'aide du logiciel IBM SPSS Statistics 19, pour déterminer si la différence inter-groupe $\left(\mathrm{CM}_{\mathrm{LM}}\right.$ vs. $\mathrm{FR}_{\mathrm{LE}} ; \mathrm{FR}_{\mathrm{LE}}$ vs. $\mathrm{FR}_{\mathrm{LM}}$ et $\mathrm{CM}_{\mathrm{LM}}$ vs. $\left.\mathrm{FR}_{\mathrm{LM}}\right)$ était significative.

\subsection{Résultats}

La figure 2 à gauche illustre la mesure des intervalles non normalisés (moyennes et écarttypes (ET) de $\Delta \mathrm{C}$ (ET des intervalles consonantiques de l'énoncé) et de $\% \mathrm{~V}$ ou proportion des intervalles vocaliques de l'énoncé) appliquées aux productions en $\mathrm{CM}_{\mathrm{LM}}$, en $\mathrm{FR}_{\mathrm{LE}}$ et en $\mathrm{FR}_{\mathrm{LM}}$. Pour toutes les métriques utilisées ici, nous notons d'emblée d'importants ET dans chaque condition linguistique. Le groupe $\mathrm{FR}_{\mathrm{LE}}$ présente des valeurs de $\Delta \mathrm{C}$ plus proches du groupe $\mathrm{FR}_{\mathrm{LM}}$ et des valeurs de $\% \mathrm{~V}$ plus proches du groupe $\mathrm{CM}_{\mathrm{LM}}$. En effet, au niveau du $\Delta \mathrm{C}$, le score des productions en $\mathrm{FR}_{\mathrm{LE}}$ est plus grand que celui en $\mathrm{CM}_{\mathrm{LM}}$ et celui en $\mathrm{FR}_{\mathrm{LM}}$. $\mathrm{La}$ différence entre les productions en $\mathrm{FR}_{\mathrm{LE}}$ et en $\mathrm{CM}_{\mathrm{LM}}$ est significative $\left(\mathrm{t}_{198}=-8,6 ; \mathrm{p}<0,001\right)$; celle entre $\mathrm{FR}_{\mathrm{LE}}$ et $\mathrm{FR}_{\mathrm{LM}}$ ne l'est pas $\left(\mathrm{t}_{148}=0,5 ; \mathrm{p}=0,591\right)$; la différence entre les productions en $\mathrm{CM}_{\mathrm{LM}}$ et en $\mathrm{FR}_{\mathrm{LM}}$ est significative $\left(\mathrm{t}_{148}=-6,3 ; \mathrm{p}<0,001\right)$.

Au niveau du $\% \mathrm{~V}$, le score des productions en $\mathrm{FR}_{\mathrm{LE}}$ se situe entre celui en $\mathrm{CM}_{\mathrm{LM}}$ et celui en $\mathrm{FR}_{\mathrm{LM}}$. La différence entre les productions $\mathrm{FR}_{\mathrm{LE}} / \mathrm{CM}_{\mathrm{LM}}$ est significative $\left(\mathrm{t}_{198}=4\right.$; $\mathrm{p}<0,001)$ comme celle entre $\mathrm{FR}_{\mathrm{LE}}$ et $\mathrm{FR}_{\mathrm{LM}}\left(\mathrm{t}_{148}=8,2 ; \mathrm{p}<0,001\right)$ ainsi que celle entre $\mathrm{CM}_{\mathrm{LM}}$ et $\mathrm{FR}_{\mathrm{LM}}\left(\mathrm{t}_{148}=10 ; \mathrm{p}<0,001\right)$.

La figure 2 à droite illustre la mesure des intervalles normalisés (coefficient de variation $\mathrm{du} \Delta \mathrm{C} \operatorname{Varco} \Delta \mathrm{C}$ et coefficient de variation du $\Delta \mathrm{V} \operatorname{Varco} \Delta \mathrm{V})$. Concernant $\operatorname{Varco} \Delta \mathrm{C}$, le score du groupe $\mathrm{FR}_{\mathrm{LE}}$ se situe entre celui du groupe $\mathrm{CM}_{\mathrm{LM}}$ et celui du groupe $\mathrm{FR}_{\mathrm{LM}}$ et plus près de celui-ci. La différence $\mathrm{FR}_{\mathrm{LE}} / \mathrm{CM}_{\mathrm{LM}}$ est significative $\left(\mathrm{t}_{198}=-3,8 ; \mathrm{p}<0,001\right)$ et il n'y a pas de différence significative $\mathrm{FR}_{\mathrm{LE}} / \mathrm{FR}_{\mathrm{LM}}\left(\mathrm{t}_{148}=-1,5 ; \mathrm{p}=0,122\right)$, donc les valeurs de Varco $\Delta \mathrm{C}$ des productions en $\mathrm{FR}_{\mathrm{LE}}$, comparables à celles en $\mathrm{FR}_{\mathrm{LM}}$, sont supérieures à celles en $\mathrm{CM}_{\mathrm{LM}}$ (différence $\mathrm{CM}_{\mathrm{LM}} / \mathrm{FR}_{\mathrm{LM}}$ significative : $\mathrm{t}_{148}=-4,7 ; \mathrm{p}<0,001$ ).

Les résultats du Varco $\Delta \mathrm{V}$ montrent que le score du groupe $\mathrm{FR}_{\mathrm{LE}}$ est le plus élevé et celui du groupe $\mathrm{CM}_{\mathrm{LM}}$ est le moins élevé (différence significative $\left(\mathrm{t}_{198}=-6,4 ; \mathrm{p}<0,001\right)$ alors 
que la différence entre le groupe $\mathrm{FR}_{\mathrm{LE}}$ et le groupe $\mathrm{FR}_{\mathrm{LM}}$ ne l'est pas $\left(\mathrm{t}_{148}=1,4 ; \mathrm{p}=0,171\right)$, car les valeurs de $\operatorname{Varco} \Delta \mathrm{V}$ des productions en $\mathrm{FR}_{\mathrm{LE}}$, comparables à celles des paroles en $\mathrm{FR}_{\mathrm{LM}}$, sont supérieures à celles des paroles en $\mathrm{CM}_{\mathrm{LM}}$. D'ailleurs, la différence entre le groupe $\mathrm{CM}_{\mathrm{LM}}$ et le groupe $\mathrm{FR}_{\mathrm{LM}}$ n'est pas due au hasard $\left(\mathrm{t}_{198}=-4,1 ; \mathrm{p}<0,001\right)$.
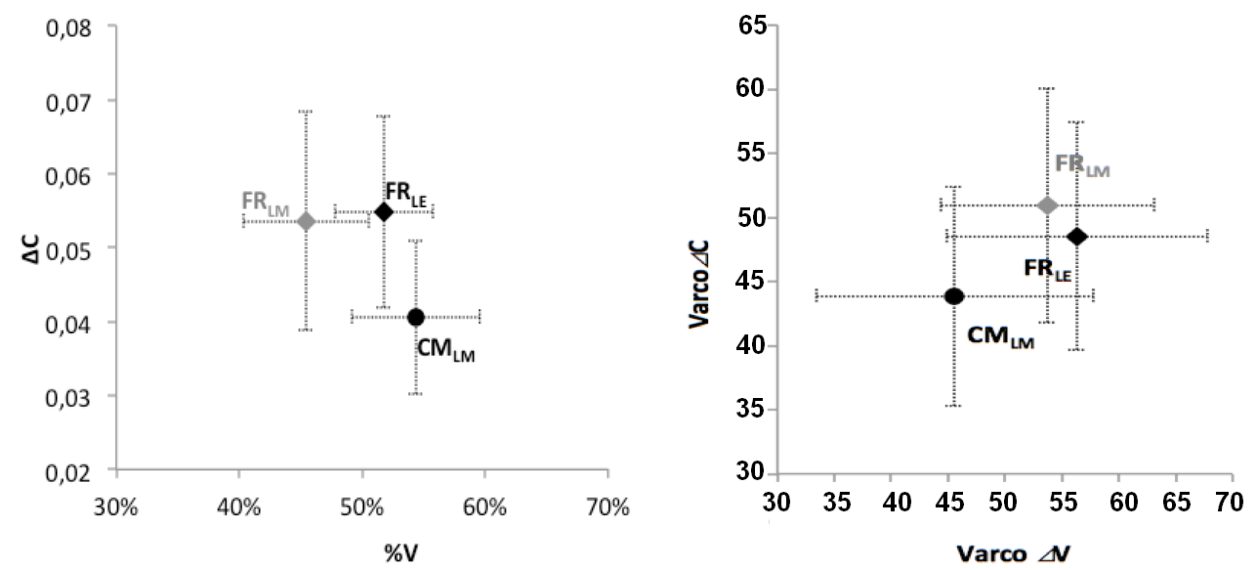

Fig. 2. Gauche : Moyenne et écart-type de la proportion des intervalles vocaliques (abscisse) et de l'écart-type des intervalles consonantiques (ordonnée) : \%V/ $\Delta \mathrm{C}$. Droite : mêmes données pour le coefficient de variation du $\Delta \mathrm{C}$ (abscisse) et le coefficient de variation du $\Delta \mathrm{V}$ (ordonnée) de nos sujets.

La figure 3 illustre la mesure des indices de variabilités par paires (PVIs) pour la variabilité brute des intervalles consonantiques successifs (rPVI-C) et la variabilité normalisée des intervalles vocaliques successifs (nPVI-V) : le groupe $\mathrm{FR}_{\mathrm{LE}}$ présente des valeurs de $\mathrm{rPVI}-\mathrm{C}$ et de nPVI-V plus proches de celles du groupe $\mathrm{FR}_{\mathrm{LM}}$ (différence pour rPVI-C entre le groupe $\mathrm{FR}_{\mathrm{LE}}$ et le groupe $\mathrm{CM}_{\mathrm{LM}}$ significative $\left(\mathrm{t}_{148}=-7 ; \mathrm{p}<0,001\right)$ et pas de différence significative entre le groupe $\mathrm{FR}_{\mathrm{LE}}$ et le groupe $\mathrm{FR}_{\mathrm{LM}}: \mathrm{t}_{148}=0,2 ; \mathrm{p}=0,872$ ).

Concernant nPVI-V, le score du groupe $\mathrm{FR}_{\mathrm{LE}}$ est le plus élevé et celui du groupe $\mathrm{CM}_{\mathrm{LM}}$ est le moins élevé. La différence $\mathrm{FR}_{\mathrm{LE}} / \mathrm{CM}_{\mathrm{LM}}$ est significative $\left(\mathrm{t}_{148}=-5,7 ; \mathrm{p}<0,001\right)$.

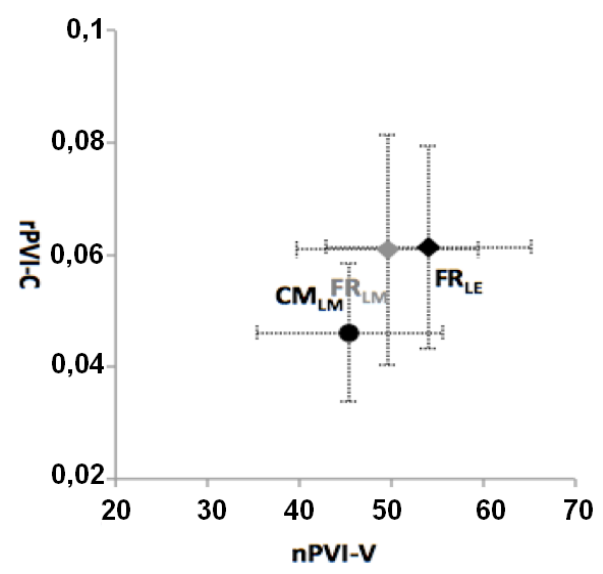

Fig. 3. Moyenne et écart-type de nPVI-V (variabilité normalisée des intervalles vocaliques successifs, abscisse) et de rPVI-C (variabilité brute des intervalles consonantiques successifs, ordonnée) appliquées aux productions en $\mathrm{CM}_{\mathrm{LM}}$, (chinois mandarin langue maternelle), $\mathrm{FR}_{\mathrm{LE}}$ (français langue étrangère) et $\mathrm{FR}_{\mathrm{LM}}$ (français langue maternelle). 


\section{Etude perceptive}

\subsection{Méthodologie}

Nous avons élaboré un test de discrimination AXB comprenant 20 triplets de stimuli, sélectionnés aléatoirement. Chaque triplet contient trois stimuli qui sont respectivement la version délexicalisée d'une phrase en $\mathrm{CM}_{\mathrm{LM}}$, d'une phrase en $\mathrm{FR}_{\mathrm{LE}}$ et d'une phrase en $\mathrm{FR}_{\mathrm{LM}}$. Ce test compte donc au total 60 stimuli $(3$ stimuli $\times 20$ triplets). Les paroles ont été délexicalisées grâce à la technique de synthèse MBROLA (Dutoit et al., 1996). La délexicalisation des productions, qui permet d'enlever les propriétés phonétiques, phonotactiques, accentuelles et intonatives et de ne préserver que les propriétés rythmiques de la parole (Ramus \& Mehler, 1999) nous a semblé un moyen plus approprié pour atteindre notre objectif du test perceptif. Cette délexicalisation, tout en conservant les mêmes durées d'intervalles vocaliques et consonantiques que celles d'origines, a consisté à normaliser l'ensemble des données à une fréquence fondamentale à $150 \mathrm{~Hz}$ d'une part, et à remplacer toutes les consonnes par/s/ et les voyelles par/a/.

Un script Praat ExperimentMFC (Multiple Forced Choice listening experiment) disponible en ligne et consulté en avril 2014 (https://searchcode.com/codesearch/view/15750616/) a été modifié pour notre test de discrimination $\mathrm{AXB}$ dont le but sous-jacent était de savoir si le rythme de $F R_{L E}(X)$ ressemblait plus au rythme de $C M_{L M}(A)$ ou au rythme de $F R_{L M}(\mathrm{~B})$. Les triplets ont été placés en ordre aléatoire et les sujets pouvaient écouter chaque stimulus « sasasa » jusque trois fois. La pause avant, après chaque stimulus et entre deux stimuli était de $2 \mathrm{~s}$.

Neuf françaises natives sans formation en mandarin, étudiantes en région parisienne, ont participé au test de perception sous Praat, toutes munies d'un casque. 20 réponses ont été obtenues par auditrice, soit 180 réponses en tout, mais nous avons exclu de nos résultats un sujet et deux réponses de deux sujets à cause du temps de réaction trop court, laissant présager une réponse aléatoire. Au total, 158 réponses ont donc été analysées.

\subsection{Résultats}

Les taux de réponses de chaque sujet au test sont présentées dans le Tableau 3 montrant la tendance générale des auditrices françaises à percevoir majoritairement le rythme du stimulus issu des productions en $\mathrm{FR}_{\mathrm{LE}}$ plutôt comme analogue à celui du $\mathrm{CM}_{\mathrm{LM}}$ : à part le Sujet 6 dont les réponses se répartissent de façon égale, le nombre de $\mathrm{CM}_{\mathrm{LM}}$ choisis par les sept sujets est significativement supérieur à celui de $\mathrm{FR}_{\mathrm{LM}}\left(\mathrm{t}_{14}=5,7 ; \mathrm{p}<0,0001\right)$. Nous pouvons donc affirmer que le transfert rythmique du CM au français dans l'apprentissage du FLE est reconnaissable pour nos locuteurs natifs du français pour notre population.

Tableau 3. Pourcentages de réponses A (rythme du $\mathrm{CM}_{\mathrm{LM}}$ ) et $\mathrm{B}$ (rythme du $\mathrm{FR}_{\mathrm{LM}}$ ), choisies par huit sujets (S) au test de perception. Une donnée a été éliminée pour les sujets 2 et 8 (voir texte)

\begin{tabular}{|c|c|c|c|c|c|c|c|c|c|c|}
\hline Stimulus X (FR $\mathrm{LM})$ perçu comme : & $\mathrm{S} 1$ & $\mathrm{~S} 2$ & $\mathrm{~S} 3$ & $\mathrm{~S} 4$ & $\mathrm{~S} 5$ & $\mathrm{~S} 6$ & $\mathrm{~S} 7$ & $\mathrm{~S} 8$ & Moyenne & ET \\
\hline A $\left(\mathrm{CM}_{\mathrm{LM}}\right)$ & 60 & 58 & 55 & 65 & 75 & 50 & 60 & 63 & $\mathbf{6 0 , 8}$ & $\mathbf{7 , 4}$ \\
\hline B $\left(\mathrm{FR}_{\mathrm{LM}}\right)$ & 40 & 42 & 45 & 35 & 25 & 50 & 40 & 37 & $\mathbf{3 9 , 2}$ & $\mathbf{7 , 4}$ \\
\hline
\end{tabular}

\section{Discussion générale et conclusion}


Selon notre hypothèse de recherche, nous nous attendions à ce que les valeurs des mesures rythmiques appliquées aux productions en $\mathrm{FR}_{\mathrm{LE}}$ se rapprochent plus de celles appliquées à la production en $\mathrm{CM}_{\mathrm{LM}}$ que de celles mesurées sur les paroles en $\mathrm{FR}_{\mathrm{LM}}$. Cependant, parmi les six mesures utilisées dans notre étude, seul $\% \mathrm{~V}$ peut confirmer l'hypothèse de départ, avec une différence significative entre le rythme du $\mathrm{FR}_{\mathrm{LE}}$ et le rythme du $\mathrm{FR}_{\mathrm{LM}}$. D'autre part, la différence rythmique entre le $\mathrm{FR}_{\mathrm{LE}}$ et le $\mathrm{CM}_{\mathrm{LM}}$ est significative d'après les valeurs des six mesures, ce qui met en doute l'incidence qu'a le rythme du chinois mandarin sur le transfert rythmique dans l'apprentissage du $\mathrm{FR}_{\mathrm{LE}}$ pour notre population. Ceci pourrait donner corps à la contestation sur l'emploi des mesures rythmiques dans l'étude du transfert rythmique de la LM à une LE (Ferjan et al., 2008). Le schéma horizontal est en effet le seul aspect du rythme qui est investigué par ces mesures rythmiques. L'aspect proéminent du rythme n'est ici pas mis en évidence (Kohler 2009).

De plus, la différence $\mathrm{CM}_{\mathrm{LM}} / \mathrm{FR}_{\mathrm{LM}}$ atteint également le seuil de significativité, ce qui peut être supposé d'après les propriétés phonologiques relatives au rythme de ces deux langues. Gabriel et al. (2014) ont montré que, dans un contexte scolaire allemand, des apprenants sinophones parlant le $\mathrm{CM}_{\mathrm{LM}}$ pouvaient plus facilement acquérir le $\mathrm{FR}_{\mathrm{LE}}$ que des germanophones natifs, car le chinois mandarin peut être positivement transféré au $\mathrm{FR}_{\mathrm{LE}}$, mais aussi à cause de facteurs extralinguistiques (attitude et conscience phonologique).

Cela montre que le rythme de la langue maternelle, selon ces paramètres acoustiques, n'interfère pas, pour notre population, sur la production du rythme du français par ces apprenants sinophones du FR $\mathrm{LE}_{\mathrm{LE}}$. Dans cette optique, ils « se débarrassent » de l'influence du rythme du chinois mandarin lors de la production en $\mathrm{FR}_{\mathrm{LE}}$. Cependant, ces apprenants ont un niveau intermédiaire avancé : des mesures analogues avec des débutants seraient souhaitables afin de savoir si le niveau de français interfère avec ce transfert, car « les effets d'influence translinguistique peuvent intervenir dans la progression de l'apprentissage et se résorber lorsque l'apprenant devient plus compétent dans la L2» (Monville-Burston, 2013).

Ces résultats sont cependant à relativiser en fonction de la variabilité des mesures obtenues pour chaque individu, comme l'illustre le tableau $4:$ les mesures des productions des sujets sinophones $n^{\circ} 1$ (séjournant en France depuis peu) et $n^{\circ} 2$ pour lesquelles notre hypothèse d'un transfert du $\mathrm{CM}_{\mathrm{LM}}$ vers le $\mathrm{FR}_{\mathrm{LE}}$ est attestée acoustiquement, concernent respectivement 3 et 4 métriques rythmiques, à l'inverse du sujet 6 pour lequel aucune métrique acoustique ne valide notre hypothèse, et des sujets 3, 5, 7 et 10 dont une seule mesure rythmique atteste notre supposition initiale.

Tableau 4. Sujets (numérotés) dont notre hypothèse de valeurs voisines entre le $\mathrm{FR}_{\mathrm{LE}}$ (Français langue étrangère) et le $\mathrm{CM}_{\mathrm{LM}}$ (Chinois mandarin langue maternelle) est confirmée (cellules grisées), pour chaque mesure rythmique.

\begin{tabular}{|c|c|c|c|c|c|c|c|c|c|c|}
\hline$\% \mathrm{~V}$ & 1 & 2 & 3 & 4 & 5 & 6 & 7 & 8 & 9 & 10 \\
\hline$\Delta \mathrm{C}$ & 1 & 2 & 3 & 4 & 5 & 6 & 7 & 8 & 9 & 10 \\
\hline Varco $\Delta \mathrm{C}$ & 1 & 2 & 3 & 4 & 5 & 6 & 7 & 8 & 9 & 10 \\
\hline Varco $\Delta \mathrm{V}$ & 1 & 2 & 3 & 4 & 5 & 6 & 7 & 8 & 9 & 10 \\
\hline nPVI-V & 1 & 2 & 3 & 4 & 5 & 6 & 7 & 8 & 9 & 10 \\
\hline rPVI-C & 1 & 2 & 3 & 4 & 5 & 6 & 7 & 8 & 9 & 10 \\
\hline
\end{tabular}

En revanche, notre test de discrimination a montré que les auditeurs natifs du français tendent à percevoir le rythme du $\mathrm{FR}_{\mathrm{LE}}$ comme ressemblant majoritairement à celui du $\mathrm{CM}_{\mathrm{LM}}$. En d'autres termes, le rythme du $\mathrm{FR}_{\mathrm{LE}}$ n'est pas considéré comme rythme « natif » pour les auditeurs natifs du français. 
Or, nos résultats perceptifs sont en contradiction avec nos analyses acoustiques où le transfert rythmique n'est pas évident d'après les valeurs des mesures rythmiques, ce qui pourrait s'expliquer soit par la relation non-linéaire entre l'acoustique et la perception en termes de rythme (Roach 1982), soit par l'emploi inapproprié des mesures pour l'étude de ce transfert rythmique. Toutefois, cette disparité entre les résultats de nos deux expériences peut aussi s'expliquer par le fait que nous ne savons pas exactement sur quelle différence les auditeurs ont forgé leur jugement, puisque nous ne leur avons pas demandé de se focaliser sur le rythme d'une part, ni n'avons testé la cohérence intralocuteur en dupliquant certains triplets d'autre part. En outre, malgré la délexicalisation, quelques propriétés segmentales comme les durées des /s/ peuvent subsister et être à l'origine de certaines différences ou proximités perçues. De plus, il n'est pas possible d'estimer totalement s'il y a corrélation entre mesure acoustique des productions d'un sujet sinophone donné, et perception de sa production par les français natifs, car nous ne savons pas à quel locuteur $\mathrm{du}$ chinois mandarin correspondent les stimuli délexicalisés en $\mathrm{CM}_{\mathrm{LM}}$ et en $\mathrm{FR}_{\mathrm{LE}}$ d'un triplet donné. Enfin, une autre limite de nos investigations tient à certaines importantes différences de durées entre deux phrases d'un même triplet: les auditeurs peuvent alors avoir perçu une différence de durée globale et non de rythme.

Finalement, n'oublions pas que la performance des apprenants sinophones en rythme du français peut éventuellement découler de l'interférence conjointe de leur langue maternelle et de leur seconde langue apprise - l'anglais - qui est le prototype des langues à isochronie accentuelle.

Le transfert rythmique du chinois mandarin au $\mathrm{FR}_{\mathrm{LE}}$, n'a, à notre connaissance, fait l'objet que d'une publication (Gabriel et al. 2014). Ce transfert n'est avéré pour nos apprenants sinophones du $\mathrm{FR}_{\mathrm{LE}}$, de niveau intermédiaire, véritablement que pour la variable $\% \mathrm{~V}$, et perceptivement. Il conviendrait de s'intéresser ultérieurement à ce transfert pour des débutants d'une part, et de considérer l'aspect proéminent du rythme d'autre part.

Ce travail a bénéficié d'une aide du LabEx EFL (ANR-10- LABX-0083).

\section{Références bibliographiques}

Abercrombie, D. (1967). Elements of General Phonetics. Edinburgh University Press : Edinburgh.

Angoujard, J-P. (1997). Théorie de la syllabe : rythme et qualité. CNRS Éditions : Paris.

Arvaniti, A. (2009). Rhythm, Timing and the Timing of Rhythm. Phonetica, 66(1-2), 46-63.

Astésano, C. (2001). Rythme et accentuation en français: invariance et variabilité stylistique. L'Harmattan : Paris.

Best, C. (1995). A Direct Realist View of Cross-language Speech Perception, in: Strange, W. (éd), Speech Perception and Linguistic Experience: Issues in Cross-language Research, 171-204, York Press : Timonium.

Boersma, P., Weenink, D. (2013). Praat: Doing phonetics by computer, logiciel téléchargé le 7 janvier $2013 \mathrm{du}$ site Internet [http://www.fon.hum.uva.nl/praat/].

Borrell, A. (1996). Parallèle entre perception et production? Complexité du lien entre reconnaissance et production des unités phonético-phonologiques. La Linguistique, 32(2), 105-116.

Brown, A. (1988). The staccato effect in the pronunciation of English in Malaysia and Singapore, in: Foley, J. (éd), New Englishes - the Case of Singapore, 115-128, Singapore University Press.

Chao, Y-R. (1968). A Grammar of Spoken Chinese. University of California Press : Berkeley.

Chevallier-Wixler, D., Dupleix, D., Jouette, I., Mègre, B. (2007). Réussir le Dalf, Niveaux C1 et C2 du Cadre européen commun de référence. Didier : Paris.

Dauer, R.M. (1983). Stress-timing and syllable-timing reanalyzed. Journal of Phonetics, 11, 51-62. 
Dellwo, V. (2006). Rhythm and speech rate: A variation coefficient for $\Delta$ C. Language and languageprocessing, 231-241.

Di Cristo, A., Hirst, D.J. (1997). L'accentuation non emphatique en français, in : Perrot, J. (éd), Polyphonie pour Ivan Fónagy, l'Harmattan, 71-101.

Di Cristo, A. (2013). La prosodie de la parole. De Boeck Solal : Bruxelles.

Di Cristo, A. (2016). Les musiques du français parlé. De Gruyter : Berlin.

Duanmu, S. (2007). The Phonology of Standard Chinese. Oxford University Press : New York.

Duez, D. (2007). Prosodie et rythme, in : Auzou P. et al. (éd), Les dysarthries, SOLAL, 181-188.

Dutoit, T., Pagel, V., Pierret, N., Bataille, F., Van der Vrecken, O. (1996). The MBROLA project: Towards a set of highquality speech synthesizers free of use for non commercial purposes, Spoken Language-ICSLP 96, Philadelphia, 3, 1393-1396.

Ellis, R. (1994). The study of second language acquisition. Oxford University Press : Oxford.

Ferjan, N., Ross, T., Arvaniti, A. (2008). Second language rhythm and rhythm metrics. Journal of the Acoustical Society of America, 123, 3427.

Flege, J.E. (1995). Second language speech learning: Theory, findings, and problems, in Strange, W. (éd), Speech Perception and Linguistic Experience: Issues in cross-language research, 233-277, York Press : Timonium.

Fraisse, P. (1956). Les Structures rythmiques : étude psychologique. Publications universitaires de Louvain : Louvain.

Gabriel, C., Stahnke, J., \& Thulke, J. (2014). On the acquisition of French speech rhythm in a multilingual classroom: Evidence from linguistic and extra-linguistic data. Congrès Mondial de Linguistique Française CMLF 2014, In SHS Web of Conferences (Vol. 8, pp. 1267-1283). EDP Sciences.

Grabe, E., \& Low, E.L. (2002). Durational Variability in Speech and the Rhythm Class Hypothesis, in: Gussenhover, C., Warner, N. (éd), Papers in Laboratory Phonology 7, 515-546, Berlin.

Gut, U. (2003). Non-native speech rhythm in German. Proceedings of the $15^{\text {th }}$ International Congress of Phonetic Sciences, Barcelone : 2437-2440.

Kober-Kleinert, C., Parizet, M-L., Poisson-Quinton, S. (2007). Activités pour le Cadre Européen Commun de Référence, Niveaux C1-C2. CLE INTERNATIONAL : Paris.

Kohler, K.J. (2009). Rhythm in Speech and Language. Phonetica, 66(1-2), 29-45.

Kuhl, P.K., Conboy, B.T., Coffey-Corina, S., Padden, D., Rivera-Gaxiola, M., \& Nelson, T. (2008). Phonetic learning as a pathway to language: new data and native language magnet theory expanded (NLM-e). Philosophical Transactions of the Royal Society B: Biological Sciences, 363(1493), 979-1000.

Ladefoged, P. (1975). A Course in Phonetics. Harcourt Brace Jovanovich : New York.

Lado, R. (1957). Linguistics across culture: Applied linguistics for language teachers. University of Michigan : Ann Arbor.

Lai, Yi-hsiu. (2011). An Acoustic Study of Mandarin Rhythm in Taiwan. Proceedings of the $17^{\text {th }}$ International Congress of Phonetic Sciences, Hongkong, 1166-1169.

Léon, P. (2011). Phonétisme et prononciations du français. Armand Colin : Paris.

Lété, B. (2008). La consistance phonographique: une mesure statistique de la complexité orthographique, in: Brissaud, C., Jaffré, J.-P., Pellat, J.-C. (éd), Nouvelles recherches en orthographe. Lambert-Lucas : Limoges.

Lin, H., Wang, Q. (2007). Mandarin rhythm: An acoustic study. Journal of Chinese Language and Computing, 17(3), 127-140.

Liu, X-Q. (2003). Analyses du rythme en chinois moderne. Thèse pour l'obtention du grade de docteur de Beijing University, Beijing. 
Low, E.L., Grabe, E., Nolan, F. (2000). Quantitative characterizations of speech rhythm: 'syllabletiming' in Singapore English. Language and Speech, 43(4), 377-401.

Mairano, P. (2010). Rhythm prosody: Acoustic and perceptive studies, Thèse pour l'obtention du grade de docteur de l'Université de Turin, Turin.

Mok, P. P-K., Dellwo V. (2008). Comparing native and non-native speech rhythm using acoustic rhythmic measures: Cantonese, Beijing Mandarin and English. Proceedings of Speech Prosody, 423-426.

Mok, P. P-K. (2009). On the syllable-timing of Cantonese and Beijing Mandarin. Chinese Journal of Phonetics, 2, 148-154.

Monville-Burston, M. (2013). Complexité et transfert dans l'acquisition du français langue étrangère : le cas des apprenants chypriotes du FLE. Travaux de linguistique 2013/1 (nº6), 97-134

Pike, K.L. (1945). The Intonation of American English. University of Michigan Press : Ann Arbor.

Ramus, F. (1999a). Rythme des langues et acquisition du langage, Thèse pour l'obtention du grade de docteur de L'EHESS, École des Hautes Études en Sciences Sociales : Paris.

Ramus, F. (1999b). La discrimination des langues par la prosodie: Modélisation linguistique et études comportementales, in: Pellegrino, F. (éd), De la caractérisation à l'identification des langues, Actes de la $1^{\text {ère }}$ journée d'étude sur l'identification automatique des langues, Lyon, Éditions de 1'Institut des Sciences de 1'Homme, 186-201.

Ramus, F., Mehler, J. (1999). Language identification with suprasegmental cues: A studybased on speech resynthesis. Journal of the Acoustical Society of America, 105(1), 512-521.

Ramus, F., Nespor, M., Mehler, J. (1999). Correlates of Linguistic Rhythm in the Speech Signal. Cognition, 73(3), 265-292.

Ramus, F. (2002). Acoustic correlates of linguistic rhythm: Perspectives. Proceedings of Speech Prosody 2002, 155-120, Aix-en-Provence.

Rasier, L., Hiligsmann, P. (2007). Prosodic transfer from L1 to L2. Theoretical and methodological issues. Nouveaux cahiers de linguistique française, 28, 41-66.

Renard, R. (1979). Introduction à la méthode verbo-tonale de correction phonétique. Didier : Paris.

Renard, R. (2002). Apprentissage d'une langue étrangère/seconde : 2. La phonétique verbo-tonale. De Boeck Université : Bruxelles.

Roach, P. (1982). On the distinction between 'stress-timed' and 'syllable-timed' languages, in: Crystal, D. (éd), Linguistic controversies, 73-79, Edward Arnold : London.

Schwartz, J.-L., Basirat, A., Ménard, L., Sato, M. (2012). The Perception for Action Control Theory (PACT): a perceptuo-motor theory of speech perception. Journal of Neurolinguistics, 25(5), 336354.

Troubetzkoy, N.S. (1938, 1976). Principes de phonologie. Klincksieck : Paris.

Wang, H-J. (2004). On the Metrical Type of Modern Standard Chinese-A Type Based on Looseness. Linguistic Sciences, 3(3), 21-28.

Wenk, B-J., Wioland, F. (1982). Is French really syllable-timed ? Journal of Phonetics, 10, 193-216.

Wenk, B-J. (1985). Speech rhythms in second language acquisition. Language and Speech, 28, 157175.

White, L., Mattys, S.L. (2007a). Calibrating rhythm: First language and second language studies. Journal of Phonetics, 35, 501-522.

White, L., Mattys, S.L. (2007b). Rhythmic typology and variation in first and second languages, in Prieto, P., Mascaró, J., Solé, M-J. (éd), Segmental and prosodic issues in romance phonology, John Benjamins Publishing Company, xvi, 237-257.

Wioland, F. (1991). Prononcer les mots du français : des sons et des rythmes. Hachette FLE : Paris. 